\title{
Effect of ashwagandha (Withania Somnifera) consumption on the selected physical fitness variables of male sprinters
}

\author{
Satpal YADAV
}

Lakshmibai National Institute of Physical Education, NERC, Guwahati, Assam, India (e-mail: satpalresearch@gmail.com).

\begin{abstract}
The purpose of the study was to investigate the effect of ashwagandha (Withania Somnifera) consumption on the selected physical fitness variables of male sprinters. The total number of 20 male sprinters which equally divided 10 in each group i.e. experimental and control groups. The sample was belong to, who had participated at inter college level under Lakshmibai National Institute of physical education, North east regional center in the age group of 18 to 25 years. Ashwagandha medicine gives to 10 experimental groups for 12 week thrice a week of alternate days with milk. After the 12 weeks physical fitness test was taken on 20 male sprinters, experimental as well as control groups. In conclusion; keeping the results and discussion in view the conclusions drawn that the significant effect of Ashwagandha on sprinters related to their standing broad jump (meters), 50 yard dash (seconds), pull-ups (minutes), sit -ups (minutes), shuttle run (second), 12- min. run/walk.

Keywords: Ashwagandha, physical fitness, sprinters.
\end{abstract}

\section{INTRODUCTION}

Ashwagandha is traditionally used for general strengthening and recovery. Botanical name of ashwgandha is withania somnifera and it belongs to family solanaceae. Mainly its dried mature roots are used for medicnal purpose. The main constituents of ashwagandha are alkaloids and steroidal lactones. The major biochemical constituents of ashwagandha root are steroidal alkaloids and steroidal lactones in a class of constituents called withanolides; at present 12 alkaloids, 35 withanolides and several sitoindosides from this plant have been isolated and studied. Ashwagandha and ginseng the aqueous suspensions of roots of an Indian drug ashwagandha and the Korean drug ginseng were tested comparatively for 2 pharmacological activities, namely the anti-stress activity by the mice swimming endurance test and anabolic activities by noting gain in body weights levator ani muscle in rats (4). The supplementation of herbal medicine named shilpax forte in the players which in turn enhance the performance (2). Pharmacological and metabolic effects of ahwagandha (withania somnifera (solanaceae)) used in ayurveda as an herbal tonic and health food were studied (3). Handball enthusiasts have had more difficulty in importing their sport to the Americas. It has caught on in a few countries - notably Brazil and Argentina - but remains a curiosity in the United States, where it is often confused with the racquetball-style recreational sport of the same name.

\section{MATERIALS AND METHODS}

Study Design and Setting: Simple random sampling technique was used to select the sample. The total number of 20 male sprinters which equally divided 10 in each group i.e. experimental and control groups. The sample was belong to, who had participated at inter college level under Lakshmibai National Institute of physical education, North east regional center in the age group of 18 to 25 years.

Sampling tools: The following physical fitness test items was used for data collection for this study was Standing broad jump (Meters), 50 yard dash (Seconds)(6), Pull-ups (Minutes), Sit -ups (Minutes), Shuttle run ( $4^{*} 10$ yard) ( Second )(5), $12 \mathrm{~min}$. run/walk (Minutes) (1).

Researcher was took the sample of 20 male sprinters. Pre-test on 10 male sprinters then divided into two groups 10 experimental and 10 control groups. After the pre-test body weight was taken of experimental group, those fall in between 50 to $59 \mathrm{~kg}$ weight 
category they were taken Ashwagandha 2.5gm and those lie above $60 \mathrm{~kg}$ weight category they were taken Ashwagandha 3gm. Ashwagandha medicine give to 10 experimental groups for 12 week thrice a week of alternate days with milk. After the 12 weeks physical fitness test was taken on 20 male sprinters, experimental as well as control groups.

Statistical Analysis: To analysis the data statistically found the significant difference between experimental and control groups mean, correlation and " $\mathrm{t}$ " test was used. (7)

\section{RESULTS}

The study was conducted to show the effect of ashwagandha (Withania Somnifera) on the selected physical fitness variables of Male sprinters. The statistical analysis of the data collected on twenty $(\mathrm{N}=20)$ male sprinters is presented. For each of the chosen variable, the results pertaining to significant difference, if any, between groups were assessed by " $t$ " test and are presented in tables:
Table 1 shows that mean of standing broad jump of consumption of ashwagandha and control group was 2.683 and 2.071 respectively. The ' $t$ ' value in case of standing broad jump of consumption of ashwagandha was 2.93. Since cal. $\mathrm{t}(=2.93)>$ tab $\mathrm{t} 0.05$ (9) (=2.26), Ho (null hypothesis) is rejected at 0.05 level of significance.

The mean of 50 Yard Dash of consumption of ashwagandha and control group was 6.27 and 6.66 respectively. The ' $t$ ' value in case of standing broad jump of consumption of ashwagandha was 2.48. Since cal. $\mathrm{t}(=2.48)>$ tab t 0.05 (9) $(=2.26)$, Ho (null hypothesis) is rejected at 0.05 level of significance.

The mean of Pull Ups of consumption of ashwagandha and control group was 9.8 and 9.13 respectively. The ' $t$ ' value in case of standing broad jump of consumption of ashwagandha was 2.44. Since cal. $\mathrm{t} \quad(=2.44)>$ tab t 0.05 (9) $(=2.26)$, Ho (null hypothesis) is rejected at 0.05 level of significance.

\begin{tabular}{|c|c|c|c|c|c|}
\hline Variables & Group & Number & Mean & Pearson Correlation & 't' Value \\
\hline \multirow[t]{2}{*}{ Standing Broad Jump } & Ashwagandha & 10 & 2.683 & 0.189 & 2.93 \\
\hline & Control & 10 & 2.071 & 0.526 & 0.70 \\
\hline \multirow[t]{2}{*}{50 Yard Dash } & Ashwagandha & 10 & 6.276 & 0.124 & 2.48 \\
\hline & Control & 10 & 6.667 & 0.146 & 1.78 \\
\hline \multirow[t]{2}{*}{ Pull Ups } & Ashwagandha & 10 & 9.8 & 0.584 & 2.44 \\
\hline & Control & 10 & 9.13 & 0.250 & 1.76 \\
\hline \multirow[t]{2}{*}{ Sit Ups } & Ashwagandha & 10 & 39.26 & 0.102 & 2.66 \\
\hline & Control & 10 & 40.26 & 0.182 & 1.46 \\
\hline \multirow[t]{2}{*}{ Shuttle Run } & Ashwagandha & 10 & 9.668 & 0.124 & 3.14 \\
\hline & Control & 10 & 9.981 & 0.458 & 0.50 \\
\hline \multirow[t]{2}{*}{12 Min. Run Walk } & Ashwagandha & 10 & 23.21 & 0.274 & 3.02 \\
\hline & Control & 10 & 24.19 & 0.332 & 0.53 \\
\hline
\end{tabular}

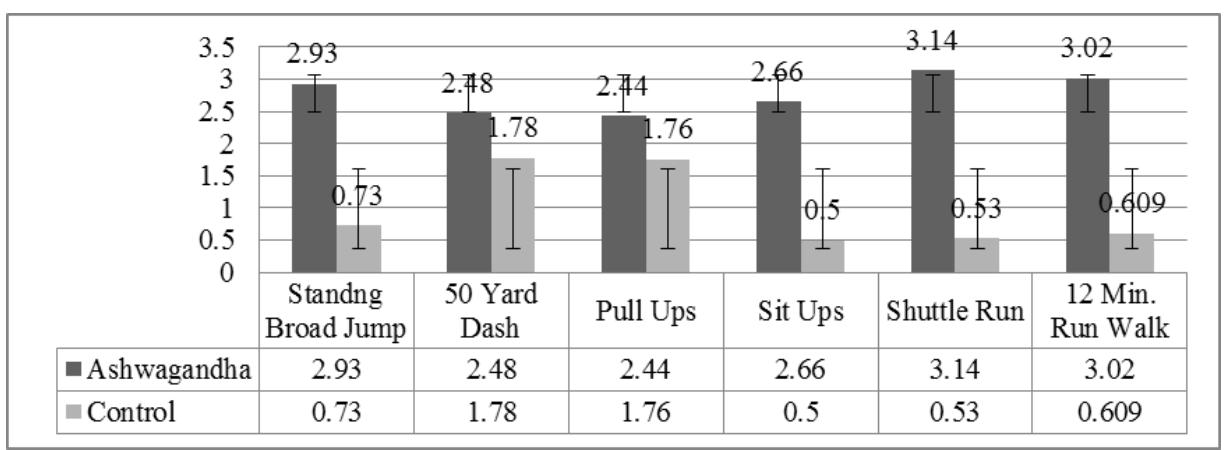

Figure 1. Physical fitness of Ashwagandha consumption and control group.

The mean of Sit Ups of consumption of ashwagandha and control group was 39.26 and 40.26 
respectively. The ' $t$ ' value in case of standing broad jump of consumption of ashwagandha was 2.66. Since cal. $\mathrm{t}(=2.66)>$ tab t 0.05 (9) $(=2.26)$, Ho (null hypothesis) is rejected at 0.05 level of significance.

The mean of Shuttle run of consumption of ashwagandha and control group was 9.66 and 9.98 respectively. The ' $t$ ' value in case of standing broad jump of consumption of ashwagandha was 3.14. Since cal. $\mathrm{t}(=3.14)>$ tab t 0.05 (9) (=2.26), Ho (null hypothesis) is rejected at 0.05 level of significance.

The mean of 12 Min. Run Walk of consumption of ashwagandha and control group was 23.21 and 24.19 respectively. The ' $t$ ' value in case of standing broad jump of consumption of ashwagandha was 3.02. Since cal. $t(=3.02)>$ tab t 0.05 (9) $(=2.26)$, Ho (null hypothesis) is rejected at 0.05 level of significance. Thus, the physical fitness of consumption of ashwagandha showed significant improvement in standing broad jump, 50 yard dash; pull ups, sit ups, shuttle run and 12 minute run walk and insignificant result in control group. As per the study the above remark can be given at $95 \%$ confidence.

\section{DISCUSSION}

The result of present study shows the significant effect of Ashwagandha on sprinters related to their Standing broad jump (Meters), 50 yard dash (Seconds) , Pull-ups (Minutes), Sit -ups (Minutes), Shuttle run (Second), $12 \mathrm{~min}$. run/walk. Ashwagandha is traditionally used for general strengthening and recovery. Strengthen to the muscular Skelton system as well making this wonder full tonic. Botanical name of Ashwagandha is withania somniferous and it belongs to family solanaceae. It is self-evident that the fit citizens are a nation's best assets and weak ones its liabilities. It is therefore the responsibility of every country to promote physical fitness of its citizens because physical fitness is the basic requirement for most of the tasks to be undertaken by an individual in his daily life. So present study show ashwagandha increase the strength and coordinate ability so there is no side effect recommended by the doctors and ashwagandha help to increase the strength and coordinative ability of the players and use for other games which requires more explosive strength, abdominal strength and coordinative ability.

In conclusion; keeping the results and discussion in view the conclusions drawn shows the significant effect of Ashwagandha on sprinters related to their Standing broad jump (Meters), 50 yard dash (Seconds), Pull-ups (Minutes), Sit -ups (Minutes), Shuttle run (Second), $12 \mathrm{~min}$. run/walk. With reference to the physical fitness between Ashwagandha consumption (experimental) and control, significant difference is noted in all diameters including standing broad jump, 50 yard dash, Pullups, Sit -ups, Shuttle run, 12 min. run/walk.

\section{REFERENCES}

1. AAPHER. Youth fitness test manual. Rev. ed., Washington, DC: American Alliance for Physical Education and Recreation, 1965.

2. Chander Munish, Ahuja Ashok and Sohal M.S. Effect of herbal Mrdicinr Shilpax forte on the Physical performance of male hockey players. Scientific Journal, sports authority of India Netaji Subash National Institute of sports Patiala, 2000; 23(3): 29-39

3. Dhuley J N. Adaptogrnic in rats and frogs. J Ethno pharmacol Pharmacology and Toxicology Section, research centre, Hindustan Antibiotics Limited, Pinmri ,Pune; India, 2000; 70 (1): 57-63

4. Gandhi A.M, Mujumdar and Patwardhan B. A comparative pharmacological investigation of Ashwgandha and Ginseng J ethnopharmacol , India Drugs research Association, Pune, 1994; 44 (3): 131-5.

5. Jensen CR, Hirst CC. Measurement in Physical Education and Athletic. New York: Macmillan publishing co. Inc, 1980.

6. Johson LB, Nelson KJ. Practical measurement for evaluation in physical education. New Delhi: Surjeet Publication, 1979.

7. Lokesh K. Methodology of Education Research. New Delhi: Vikas publishing house PVT LTD, 2007. 